
\title{
R Research Soure \\ The Relationship Between Intralobar Pulmonary Sequestration and Type a Aortic Dissection: A Case Report
}

\section{Gang-Hua Lin}

Tri-Service General Hospital https://orcid.org/0000-0002-3575-1766

Ying Yi Chen ( $\nabla$ addgujfjui@gmail.com )

Division of Thoracic Surgery, Department of Surgery, Tri-Service General Hospital, National Defense Medical Center, Taipei, Taiwan

\section{Case report}

Keywords: Intralobar pulmonary sequestration, Acute aortic dissection, Medium and large vessel vasculitis

Posted Date: March 2nd, 2021

DOI: https://doi.org/10.21203/rs.3.rs-245023/v1

License: (a) (i) This work is licensed under a Creative Commons Attribution 4.0 International License. Read Full License 


\section{Abstract}

Background: Pulmonary sequestrations often lead to serious complications such as infections, tuberculosis, fatal hemoptysis, cardiovascular problems, and even malignant degeneration, but it is rarely documented with medium and large vessel vasculitis, which is likely to result in acute aortic syndromes.

Case presentation: A 44-year-old man with a history of acute Stanford type A aortic dissection status post-reconstructive surgery five years ago. The contrast-enhanced computed tomography (CT) of the chest at that time had also revealed an intralobar pulmonary sequestration in the left lower lung region, and the angiography also presented perivascular changes with mild mural thickening and wall enhancement, which indicated mild vasculitis. The intralobar pulmonary sequestration in the left lower lung region was long-term unprocessed, which was probably associated with his intermittent chest tightness since no specific medical findings were detected but only positive sputum culture with mycobacterium avium-intracellulare complex and Aspergillus. We performed uniportal video-assisted thoracoscopic surgery with wedge resection of the left lower lung. Hypervascularity over the parietal pleura, engorgement of the bronchus due to a moderate amount of mucus, and firm adhesion of the lesion to the thoracic aorta were histopathologically noticed.

Conclusion: We hypothesized that a long-term pulmonary sequestration-related bacterial and fungal infection can result in focal infectious aortitis gradually, which may threateningly contribute to aortic dissection.

\section{Background}

Pulmonary sequestrations are associated with serious complications including bacterial and fungal infections, tuberculosis, massive hemothorax, serious hemoptysis, cardiovascular events, malignant degeneration, and even rarely but fatal medium and large vessel vasculitis. Aortitis as a form of large vessel vasculitis caused mainly by rheumatological inflammation or infection potentially results in acute aortic syndromes, including aortic dissection. We herein present a 44-year-old man with a history of acute Stanford type A aortic dissection status post-reconstructive surgery five years ago. The patient presented with a long-term unprocessed intralobar pulmonary sequestration in the left lower lung region, which was probably associated with intermittent chest tightness since no specific medical findings were detected. Furthermore, sputum culture was positive for mycobacterium avium-intracellulare complex and Aspergillus. We performed uniportal video-assisted thoracoscopic surgery with wedge resection of the left lower lung. Since the patient was relatively young and had no history of systemic hypertension, we hypothesized that a long-term pulmonary sequestration-related bacterial and fungal infection can result in focal infectious aortitis gradually, which may contribute to aortic dissection.

\section{Case Presentation}


A 44-year-old man presented to us with a history of acute aortic dissection, Stanford type A with the aortic arch and the carotid arteries involvement (Fig. 1). He had undergone reconstruction of the ascending aorta and the aortic arch up to the branching out of the right innominate artery five years ago. The contrast-enhanced computed tomography (CT) of the chest at that time had also revealed a focal area of cystic change (Fig. 2A) and patchy consolidation in the medial region of the lower lobe of the left lung. Two aberrant arteries arising from the aorta were supplying this area (Fig. 2B), which was suggestive of an intralobar pulmonary sequestration. CT angiography also revealed perivascular changes with mild mural thickening and wall enhancement, which indicated mild vasculitis (Fig. 2C). At that time, he immediately underwent emergent surgery for the aortic dissection, which could prove to be fatal, but attention was not given to the pulmonary sequestration at that time. Histopathology of ascending aorta intima revealed myxoid degeneration, intimal hyperplasia, and abundant fibrin and neutrophils.

However, a year ago, he started complaining of chest tightness, intermittently. He was admitted to the department of thoracic medicine to rule out cardiovascular or gastrointestinal pathologies, but no specific findings were revealed except for sputum culture, which was positive for mycobacterium aviumintracellulare complex and aspergillus. The patient was then admitted to the department of thoracic surgery to deal with his long-term unprocessed intralobar pulmonary sequestration. We performed uniportal video-assisted thoracoscopic surgery with an extended wedge resection of the left lower lung. Firm adhesion of the sequestrated lung to the thoracic aorta and the two aberrant arteries originating from the thoracic aorta were identified (Fig. 3A) and then resected by Endo GIA. Meanwhile, hypervascularity over the parietal pleura as well as engorgement of the bronchus were also noticed. The macroscopic findings revealed a solid pulmonary nodule about $10 \times 4 \times 2 \mathrm{~cm}^{3}$ in size with focal bronchial dilation containing hemorrhage and mucus (Fig. 3B), calcification as well as extensive necrosis of the lung. Histopathological analysis revealed small bronchial duct hyperplasia along with two lymph nodes showing reactive lymphoid hyperplasia, with no evidence of malignancy. Postoperative recovery was smooth, without any surgical complications, and the patient was discharged on the 4th postoperative day.

\section{Discussion And Conclusions}

Pulmonary sequestrations are bronchopulmonary foregut malformations, usually characterized by a nonfunctional segmental lung tissue that dissociates from the normal tracheobronchial tree or the pulmonary arteries, and commonly appears in the lower lobes of the lung. Pulmonary sequestrations lead to serious complications: fungal infections, tuberculosis (1), fatal hemoptysis, massive hemothorax, cardiovascular problems (2), and even benign or malignant degeneration. Infected pulmonary sequestration due to mycobacteria, such as Mycobacterium tuberculosis and Mycobacterium aviumintracellulare complex, occur rarely. (1) Malik et al. also demonstrated an asymptomatic intralobar pulmonary sequestration, associated with medium - and large vessel vasculitis.(3)

Aortitis, which is defined as a form of large vessel vasculitis, is characterized by the inflammation of the aortic wall. Most cases of aortitis are either due to rheumatological causes, which include large vessel 
vasculitis, giant cell arteritis and Takayasu arteritis; or because of infectious diseases, with Salmonella, Staphylococcal species, and Streptococcus pneumoniae being the most commonly identified pathogens., Tuberculosis and syphilis are rare but potentially life-threatening causes.(4) In most cases of bacterial aortitis, a segment of the aortic wall with pre-existing pathology, such as an atherosclerotic plaque or aneurysm, is seeded by the bacteria via the vasa vasorum.(5) Acute aortic syndromes, like aortic dissection and rupture, can occur in such patients with aortitis.(6) Ryder et al. presented a case of a previously healthy 39-year-old man who succumbed to aortic dissection hours after the onset of symptoms. Aortitis was detected during the postmortem examination in this case. (7) They also identified a cohort of patients who presented with a subtype of isolated inflammatory aortitis, which was characterized by aggressive vasculitis with acute inflammatory infiltration. Park et al. also described a case of an 83-year-old woman, with a history of hypertension, who arrived at the emergency department with septic shock. Stanford type A aortic dissection was revealed by the chest CT scan initially. In the end, aortitis, without giant cells and caseous necrosis, was identified, when histopathological examination of the ascending aorta was performed after the emergency surgery. (8)

Since the patient, in our case, was young and without systemic hypertension, we hypothesize that a longterm pulmonary sequestration-related bacterial and fungal infection is likely to result in infectious aortitis gradually, which can, menacingly, contribute to aortic dissection.

\section{List Of Abbreviations}

CT: Computed tomography

\section{Declarations}

Ethics approval and consent to participate: Not applicable.

Consent for publication: The patient provided written informed consent.

Availability of data and materials: Not applicable.

Competing interests: There are no any relevant competing interest.

Funding: Denied.

Authors' contributions: Lin GH and Chen YY performed the surgery, reviewed the literature and contributed to manuscript drafting. All of the authors have read and approved the final manuscript.

Acknowledgements: Not applicable.

\section{References}


1. Shiota Y, Arikita H, Aoyama K, Horita N, Hiyama J, Ono T, et al. Pulmonary sequestration associated by Mycobacterium intracellulare infection. Intern Med. 2002;41(11):990-2. Epub 2002/12/19. doi: 10.2169/internalmedicine.41.990. PubMed PMID: 12487175.

2. Fabre $\mathrm{OH}$, Porte HL, Godart FR, Rey C, Wurtz AJ. Long-term cardiovascular consequences of undiagnosed intralobar pulmonary sequestration. Ann Thorac Surg. 1998;65(4):1144-6. Epub 1998/05/16. doi: 10.1016/s0003-4975(98)00032-0. PubMed PMID: 9564949.

3. Malik S, Khurana S, Vasudevan V, Gupta N. A rare case of underlying pulmonary sequestration in a patient with recently diagnosed medium and large vessel vasculitis. Lung India. 2014;31(2):176-8. doi: 10.4103/0970-2113.129871. PubMed PMID: 24778487.

4. Gornik HL, Creager MA. Aortitis. Circulation. 2008;117(23):3039-51. doi: 10.1161/CIRCULATIONAHA.107.760686. PubMed PMID: 18541754.

5. Azar T, Berger DL. Adult intussusception. Annals of surgery. 1997;226(2):134-8. Epub 1997/08/01. PubMed PMID: 9296505; PubMed Central PMCID: PMCPmc1190946.

6. Nuenninghoff DM, Hunder GG, Christianson TJ, McClelland RL, Matteson EL. Incidence and predictors of large-artery complication (aortic aneurysm, aortic dissection, and/or large-artery stenosis) in patients with giant cell arteritis: a population-based study over 50 years. Arthritis Rheum. 2003;48(12):3522-31. Epub 2003/12/16. doi: 10.1002/art.11353. PubMed PMID: 14674004.

7. Ryder HF, Tafe LJ, Burns CM. Fatal Aortic Dissection due to a Fulminant Variety of Isolated Aortitis. JCR: Journal of Clinical Rheumatology. 2009;15(6):295-9. doi: 10.1097/RHU.0b013e3181b1246d. PubMed PMID: 00124743-200909000-00008.

8. Park BS, Min HK, Kang DK, Jun HJ, Hwang YH, Jang EJ, et al. Stanford type A aortic dissection secondary to infectious aortitis: a case report. Journal of Korean medical science. 2013;28(3):485-8. Epub 2013/03/15. doi: 10.3346/jkms.2013.28.3.485. PubMed PMID: 23486652; PubMed Central PMCID: PMCPMC3594617.

\section{Figures}



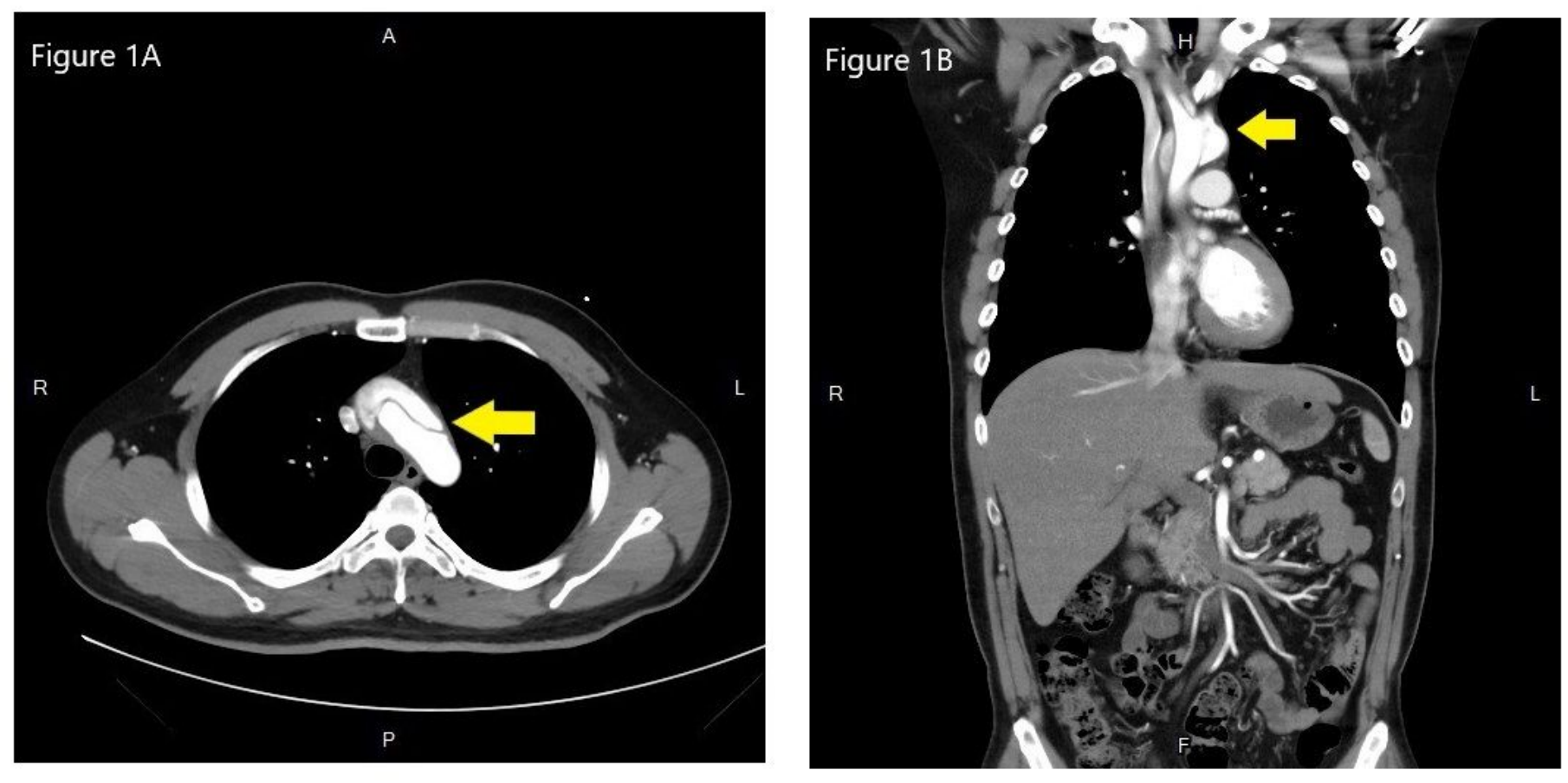

\section{Figure 1}

Type A aortic dissection with intimal flap involving the ascending aorta, the aortic arch (arrow in Fig. 1A) and the common carotid arteries bilaterally. (Fig. 1B) Arrow in Fig. 1B points to the mural thrombus in the false lumen.
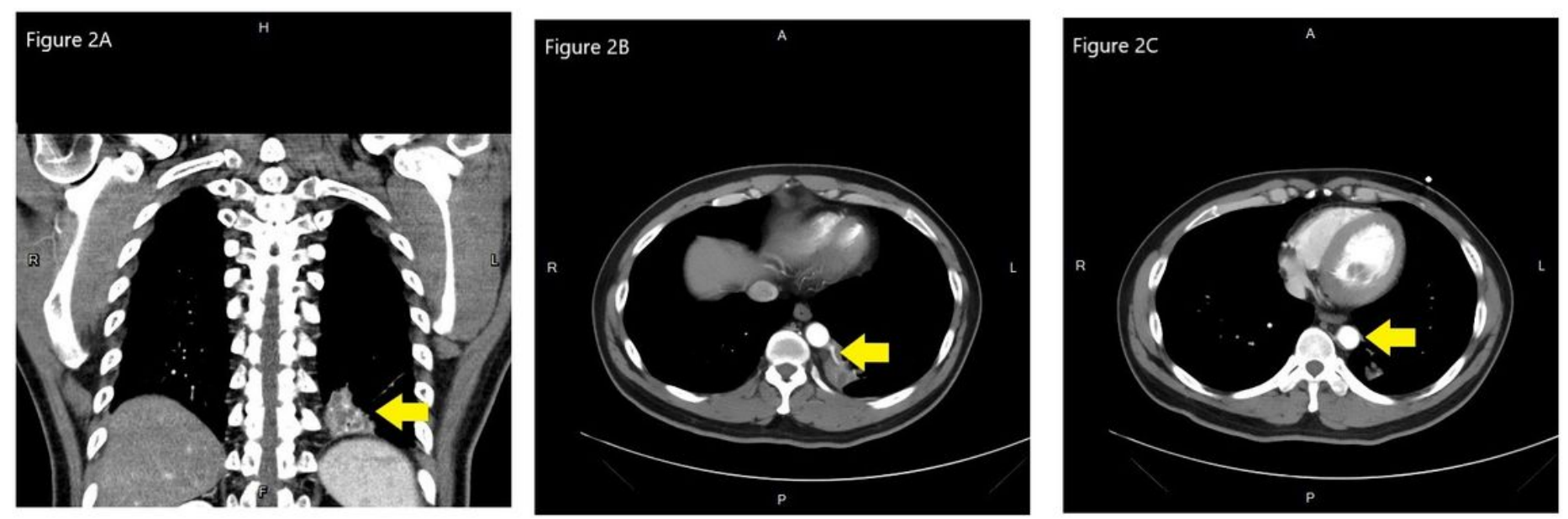

Figure 2

The contrast-enhanced computed tomography (CT) of the chest revealed a focal heterogeneous area with cystic change and patchy consolidation in the medial region of left lower lung (arrow in Fig. 2A) with two aberrant arteries (arrow in Fig 2B) arising from the thoracic aorta supplying this area. CT angiography also revealed mild mural thickening and wall enhancement which may indicate mild vasculitis (arrow in Fig. 2C) 

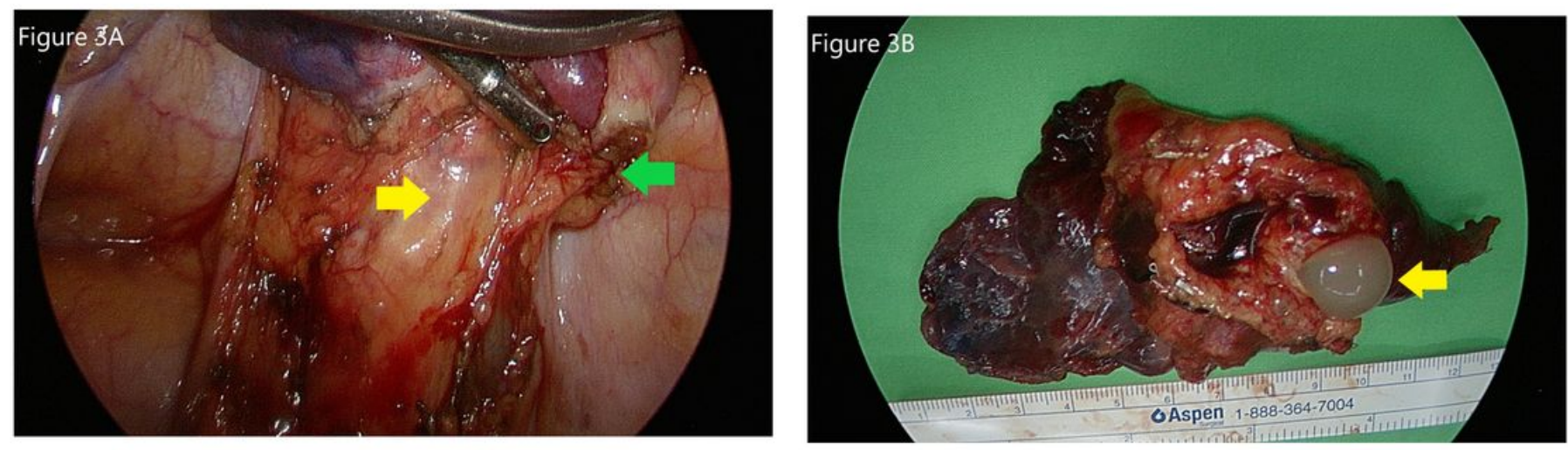

Figure 3

Video-assisted thoracoscopic surgery revealed that the two aberrant arteries (Fig. 3A, green arrow) originated from the thoracic aorta. Engorgement of the bronchus (Fig. 3A, yellow arrow) was also noticed. Gross specimen showed focal bronchial dilation that contained mucus (Fig. 3B, yellow arrow). 